\title{
PhoMT: A High-Quality and Large-Scale Benchmark Dataset for Vietnamese-English Machine Translation
}

\author{
Long Doan*, Linh The Nguyen*, Nguyen Luong Tran*, \\ Thai Hoang ${ }^{\dagger}$ and Dat Quoc Nguyen \\ VinAI Research, Hanoi, Vietnam \\ $\{$ v.longdet, v.linhnt140, v.nguyent112, v.thaihq5, v.datnq9\}evinai.io
}

\begin{abstract}
We introduce a high-quality and large-scale Vietnamese-English parallel dataset of 3.02M sentence pairs, which is $2.9 \mathrm{M}$ pairs larger than the benchmark Vietnamese-English machine translation corpus IWSLT15. We conduct experiments comparing strong neural baselines and well-known automatic translation engines on our dataset and find that in both automatic and human evaluations: the best performance is obtained by fine-tuning the pretrained sequence-to-sequence denoising autoencoder mBART. To our best knowledge, this is the first large-scale Vietnamese-English machine translation study. We hope our publicly available dataset and study can serve as a starting point for future research and applications on Vietnamese-English machine translation. We release our dataset at: https: / / github.com/VinAIResearch/PhoMT.
\end{abstract}

\section{Introduction}

Vietnam has achieved rapid economic growth in the last two decades (Baum, 2020). It is now an attractive destination for trade and investment. Due to the language barrier, foreigners usually rely on automatic machine translation (MT) systems to translate Vietnamese texts into their native language or another language they are familiar with, e.g. the global language English, so they could quickly catch up with ongoing events in Vietnam. Thus the demand for high-quality Vietnamese-English MT has rapidly increased. However, state-of-theart MT models require high-quality and large-scale corpora for training to be able to reach near humanlevel translation quality (Wu et al., 2016; Ott et al., 2018). Despite being one of the most spoken languages in the world with about $100 \mathrm{M}$ speakers, Vietnamese is referred to as a low-resource language in MT research because publicly available

\footnotetext{
${ }^{*}$ The first three authors contributed equally to this work.

${ }^{\dagger}$ Work done during internship at VinAI Research. Email: qthai912@cs.washington.edu
}

parallel corpora for Vietnamese in general and in particular for Vietnamese-English MT are not large enough or have low-quality translation pairs, including those with different sentence meaning (i.e. misalignment).

Two main concerns are detailed as follows:

- High-quality Vietnamese-English parallel corpora are either not publicly available or smallscale. Ngo et al. (2013) and Phan-Vu et al. (2019) present two corpora each comprising of $800 \mathrm{~K}$ sentence pairs, however, these two corpora are not publicly available. Thus, the VietnameseEnglish parallel corpus IWSLT15 (Cettolo et al., 2015) of $133 \mathrm{~K}$ sentence pairs extracted from TED-Talks transcripts is still considered as the standard benchmark for MT when it comes to Vietnamese. Recently, the OPUS project (Tiedemann, 2012) provides $300 \mathrm{~K}+$ sentence pairs extracted from the TED2020 v1 corpus of TEDTalks transcripts (Reimers and Gurevych, 2020).

- Larger Vietnamese-English parallel corpora are noisy, e.g. see discussions on the $300 \mathrm{~K}-600 \mathrm{~K}$ sentence pair corpora of JW300 (Agić and Vulić, 2019), OPUS's GNOME and QED (Abdelali et al., 2014) in Section 2.1, and on the OpenSubtitles corpus (Lison and Tiedemann, 2016) in Section 2.2. Recently, CCAligned (El-Kishky et al., 2020) and WikiMatrix (Schwenk et al., 2021) are created by using LASER sentence embeddings (Artetxe and Schwenk, 2019) and margin-based sentence alignment to mine parallel sentences from comparable web-document pairs. Though containing millions of Vietnamese-English parallel sentence pairs, they still have a large proportion of misalignment and low-quality translation pairs. In particular, we randomly sample from each corpus 100 sentence pairs and manually inspect their quality. We find that only 37/100 CCAligned pairs and 31/100 WikiMatrix pairs are at a high-quality translation level. 
As the first contribution, to help handle the two concerns above, we present a high-quality and large-scale Vietnamese-English parallel dataset, named PhoMT, that consists of $3.02 \mathrm{M}$ sentence pairs. Here, from PhoMT, we also prepare $38 \mathrm{~K}$ sentence pairs with manually qualitative inspection, that are used for validation and test. We believe that our dataset construction process will help develop more efficient data creation strategies for other low-resource languages. As the second contribution, we empirically investigate strong baselines on our dataset, including Transformer-base, Transformer-big (Vaswani et al., 2017) and the pre-trained sequence-to-sequence denoising autoencoder mBART (Liu et al., 2020), and compare these baselines with well-known automatic translation engines. We find that mBART obtains the highest scores in terms of both automatic and human evaluations on both translation directions. To the best of our knowledge, this is the first largescale empirical study for Vietnamese-English MT. As our final contribution, we publicly release our PhoMT dataset for research or educational purposes. We hope PhoMT together with our empirical study can serve as a starting point for future Vietnamese-English MT research and applications.

\section{Our PhoMT dataset}

Our dataset construction process consists of 4 phases. The 1st phase is to collect parallel document pairs. The 2 nd phase is a pre-processing step that is to produce cleaned and high-quality parallel document pairs and then extract sentences from these pairs. The 3rd phase is to align parallel sentences within a pair of parallel documents. The 4th phase is a post-processing step that is to filter out duplicated parallel sentence pairs and manually verify the quality of validation and test sets.

\subsection{Collecting parallel document pairs}

We collect the parallel document pairs from publicly available resources that contain original English documents and their corresponding Vietnamese-translated version. WikiHow: It is an online knowledge base of how-to guides that are available in multiple languages. We employ a multilingual WikiHow-based document summarization corpus (Ladhak et al., 2020) that contains 6616 pairs of WikiHow English articles and their Vietnamese-translated variant. TED-Talks: We use the TED2020 v1 corpus
(Reimers and Gurevych, 2020) that includes 3123 English-Vietnamese subtitle pairs of TED talks. OpenSubtitles: We employ the latest version v2018 of the OpenSubtitles corpus (Lison and Tiedemann, 2016) that contains 3886 parallel movie and TV subtitles. MediaWiki: We also use parallel documents from the MediaWiki content translation data dump. News \& Blogspot: We collect English and Vietnamese-translated versions of news and Blogspot articles from eight websites for English learners. See URLs for the described resources in the Appendix.

Here, we do not include available corpora of JW300 (Agić and Vulić, 2019), OPUS's GNOME and QED (Abdelali et al., 2014). We manually check 100 randomly sampled pairs from the $600 \mathrm{~K}$ Vietnamese-English sentence pair corpus JW300 and find that there are 71 high-quality translation pairs. However, it is worth noting that JW300 can introduce potential bias because of its religious domain. GNOME from OPUS contains 600K sentences pairs, in which most Vietnamese target sentences include many original translatable technical English words, thus not natural. QED has 340K sentence pairs, however, our investigation finds that about a half of the QED pairs are from the TEDTalks transcripts (Reimers and Gurevych, 2020); and from the remaining sentence pairs, we randomly sample 100 pairs and find that only 43 pairs have a high-quality translation.

\subsection{Pre-processing}

We find that not all of 3886 English-Vietnamese parallel document pairs in OpenSubtitles have a high-quality translation. We manually inspect each OpenSubtitles pair and remove 574/3886 (15\%) document pairs with a low-quality translation, thus remaining 3312 pairs. In MediaWiki, there are original English paragraphs appearing in some Vietnamese target documents, that have not been translated into Vietnamese yet. We employ the language identification module of fastText (Joulin et al., 2017) to identify and filter those English paragraphs out of the Vietnamese documents. We also remove reference sections and tables that appear in some MediaWiki and Blogspot documents.

To extract sentences for parallel sentence alignment in the next phase, we perform (tokenization and) sentence segmentation by using the Stanford CoreNLP toolkit (Manning et al., 2014) and RDRSegmenter (Nguyen et al., 2018) from the Vn- 


\begin{tabular}{l|l|l|l|l|l|l|l|l|l|l|l}
\hline \multirow{2}{*}{ Domain } & \multicolumn{2}{|c|}{ Total } & \multicolumn{3}{c|}{ Training } & \multicolumn{3}{c}{ Validation } & \multicolumn{3}{c}{ Test } \\
\cline { 2 - 11 } & \#doc & \#pair & \#pair & \#en/s & \#vi/s & \#pair & \#en/s & \#vi/s & \#pair & \#en/s & \#vi/s \\
\hline News & 2559 & 41504 & 40990 & 24.4 & 32.0 & 257 & 22.3 & 30.3 & 257 & 26.8 & 34.5 \\
Blogspot & 1071 & 93956 & 92545 & 25.0 & 34.6 & 597 & 26.4 & 37.8 & 814 & 23.7 & 31.5 \\
TED-Talks & 3123 & 320802 & 316808 & 19.8 & 23.8 & 1994 & 20.0 & 24.6 & 2000 & 22.0 & 27.9 \\
MediaWiki & 38969 & 496799 & 490505 & 26.0 & 32.8 & 3024 & 25.3 & 32.3 & 3270 & 27.0 & 33.7 \\
WikiHow & 6616 & 513837 & 507379 & 18.9 & 22.4 & 3212 & 17.9 & 21.5 & 3246 & 17.5 & 21.5 \\
OpenSub & 3312 & 1548971 & 1529772 & 9.7 & 11.1 & 9635 & 9.5 & 10.7 & 9564 & 10.0 & 11.4 \\
\hline All & 55650 & 3015869 & 2977999 & 15.7 & 19.0 & 18719 & 15.3 & 18.7 & 19151 & 16.2 & 19.8 \\
\hline
\end{tabular}

Table 1: Our dataset statistics. "\#doc", “\#pair", "\#en/s" and "\#vi/s" denote the number of parallel document pairs, the number of aligned parallel sentence pairs, the average number of word tokens per English sentence and the average number of syllable tokens per Vietnamese sentence, respectively. "OpenSub” abbreviates OpenSubtitles.

CoreNLP toolkit (Vu et al., 2018) for English and Vietnamese, respectively.

\subsection{Aligning parallel sentence pairs}

To align parallel sentences within a parallel document pair, our approach first employs the strong neural MT engine Google Translate to translate each English source sentence into Vietnamese. Then we use three toolkits of Hunalign (Varga et al., 2007), Gargantua (Braune and Fraser, 2010) and Bleualign (Sennrich and Volk, 2011) to perform sentence alignment via alignment between the Google-translated variants of the English source sentences and the Vietnamese target sentences. Finally, we only select sentence pairs that are aligned by at least two out of three toolkits as the output of our alignment process.

The quality of our sentence alignment output is shown in Section 2.4. Here, we discuss alignment coverage rates. On the same TED2020 v1 corpus, the automatic alignment approach OPUS (Tiedemann, 2012), based on word alignments and phrase tables, aligns a total of 326K Vietnamese sentences, ${ }^{1}$ while our approach aligns $350 \mathrm{~K}$ Vietnamese ones (i.e. a 7.5\% relative improvement). ${ }^{2}$

Note that from each resource domain except OpenSubtitles, our approach selects $99+\%$ of Vietnamese sentences to be included in the output of our alignment process. Particularly, a total of $14.6 \mathrm{~K}$ Vietnamese sentences (i.e. $0.86 \%$ ) are not selected from five resource domains of News, Blogspot, TED-Talks, MediaWiki and WikiHow. When it comes to OpenSubtitles, the rate reduces to $95 \%$ (here, 120K Vietnamese sentences are not included in our alignment output). On average, the align-

\footnotetext{
${ }^{1}$ https: / / object.pouta.csc.fi/ OPUS-TED2020/v1/moses/en-vi.txt.zip wherein duplicate removal is not performed.

${ }^{2}$ See the Appendix for an additional discussion.
}

ment coverage rate for English is about 2\% absolute lower than the one for Vietnamese as there are English source sentences that are not translated to Vietnamese in the collected corpora.

\subsection{Post-processing}

On the alignment output from the previous phase, we normalize punctuations, remove all duplicate sentence pairs within and across all document pairs, and also remove sentence pairs where the reference English sentence contains either only a single word token or swear words. ${ }^{3}$ Then we randomly split each domain into training/validation/test sets on document level with a 98.75/0.60/0.65 ratio, resulting in 2977999 training, 18876 validation and 19291 test sentence pairs.

To qualify our dataset, we manually inspect each sentence pair in the validation and test sets. Here, each pair is inspected by two out of the first three co-authors independently: one inspector checks about $(18876+19291) \times 2 / 3=25 \mathrm{~K}$ sentence pairs in 125 hours on average (i.e. 200 sentences/hour). After cross-checking, we find that in the validation set, 32 sentence pairs $(0.17 \%)$ are misaligned (i.e. completely different sentence meaning or partly preserving the sentence meaning); and 125 pairs $(0.66 \%)$ are low-quality translation ones (i.e. mostly or completely preserving the sentence meaning, however, the Vietnamese target sentence is not naturally smooth). In the test set, there are 27 misaligned sentence pairs $(0.14 \%)$ and 113 low-quality translation pairs $(0.59 \%)$. Note that performing a similar manual inspection on the training set is beyond our current human resource; however, with small proportions of misalignment and low-quality

\footnotetext{
${ }^{3}$ Regarding removals, punctuations are not taken into identifying sentence pair duplication or computing sentence length. On OpenSubtitles, we also remove sentence pairs where the reference English sentence consists of two tokens.
} 


\begin{tabular}{|c|c|c|c|c|c|c|c|c|c|c|}
\hline \multirow{3}{*}{ Model } & \multicolumn{4}{|c|}{ Validation set } & \multicolumn{6}{|c|}{ Test set } \\
\hline & \multicolumn{2}{|c|}{ En-to-Vi } & \multicolumn{2}{|c|}{ Vi-to-En } & \multicolumn{3}{|c|}{ En-to-Vi } & \multicolumn{3}{|c|}{ Vi-to-En } \\
\hline & TER $\downarrow$ & BLEU $\uparrow$ & TER $\downarrow$ & BLEU $\uparrow$ & TER $\downarrow$ & BLEU $\uparrow$ & Human $\uparrow$ & TER $\downarrow$ & BLEU $\uparrow$ & Human $\uparrow$ \\
\hline Google Translate & 45.86 & 40.10 & 44.69 & 36.89 & 46.52 & 39.86 & $23 / 100$ & 45.86 & 35.76 & $10 / 100$ \\
\hline Bing Translator & 45.36 & 40.82 & 45.32 & 36.61 & 46.04 & 40.37 & $14 / 100$ & 46.09 & 35.74 & $15 / 100$ \\
\hline Transformer-base & $4 \overline{2} . \overline{7} \overline{7}$ & $\overline{43.01}$ & ${ }^{-} 4 \overline{3} . \overline{4} \overline{2}$ & 38.26 & $\overline{4} \overline{3} . \overline{7} 9^{-}$ & $\overline{42.12}$ & $\overline{13} / 10 \overline{0}$ & 44.28 & $3 \overline{7} . \overline{1} 9^{-}$ & $\overline{13} / \overline{100}$ \\
\hline Transformer-big & 42.13 & 43.75 & 43.08 & 39.04 & 43.04 & 42.94 & $18 / 100$ & 44.06 & 37.83 & $28 / 100$ \\
\hline mBART & 41.56 & 44.32 & 41.44 & 40.88 & 42.57 & 43.46 & $32 / 100$ & 42.54 & 39.78 & $34 / 100$ \\
\hline
\end{tabular}

Table 2: Overall results. Each TER/BLEU score difference between two models is statistically significant (p-value $<0.05$ based on bootstrap resampling), except Google Translate and Bing Translator for Vi-to-En w.r.t. BLEU.

translation on the validation and test sets at the sentence pair level, we believe that our training set attains a high-quality standard. Lastly, we remove those $32+125+27+113=297$ pairs, resulting in 18719 validation and 19151 test sentence pairs of high quality for final use.

\section{Experiments}

\subsection{Experimental setup}

We conduct experiments on our PhoMT dataset to study: (i) a comparison between the wellknown automatic translation engines (here, Google Translate and Bing Translator) and strong neural MT baselines, and (ii) the usefulness of pre-trained sequence-to-sequence denoising autoencoder. In particular, we use the baseline models: Transformer-base, Transformer-big (Vaswani et al., 2017), and the pre-trained denoising auto-encoder mBART (Liu et al., 2020). We report standard metrics TER (Snover et al., 2006) and BLEU (Papineni et al., 2002), in which lower TER and higher BLEU indicate better performances. We compute the case-sensitive BLEU score using SacreBLEU (Post, 2018). See the Appendix for implementation details. Here, we select the model checkpoint that obtains the highest BLEU score on the validation set to apply to the test set.

\subsection{Automatic evaluation results}

Table 2 presents TER and BLEU scores obtained by the automatic translation engines and the neural baselines on the validation and test sets for the English-to-Vietnamese (En-to-Vi) and Vietnameseto-English (Vi-to-En) translation setups. Clearly, the baselines obtain significantly better TER and BLEU scores than the automatic translation engines for both En-to-Vi and Vi-to-En setups on both validation and test sets. It is likely because Google Translate and Bing Translator are trained on other parallel resources. Here, Transformer models obtain $1.5+$ points absolute better than both

\begin{tabular}{|c|c|c|c|c|c|c|}
\hline \multirow{2}{*}{ Model } & \multicolumn{6}{|c|}{ Vi-to-En } \\
\hline & News & BloS & TedT & MedW & WikH & OpeS \\
\hline Google Translate & 34.33 & 26.71 & 34.03 & 48.81 & 28.76 & 28.63 \\
\hline & 35.05 & 25.91 & 32.03 & 50.29 & 24.78 & 30.56 \\
\hline Transformer-base & $\overline{3} \overline{4.94}$ & $\overline{27.13}$ & $\overline{33.46}$ & $\overline{50 . \overline{5}} \bar{\sigma}^{-}$ & $\overline{28.3 \overline{7}}$ & $\overline{3} 2 . \overline{29}$ \\
\hline Transformer-big & 36.14 & 28.26 & 34.46 & 51.20 & 28.86 & 32.69 \\
\hline mBART & 37.04 & 29.26 & 36.43 & 53.93 & 30.63 & 34.13 \\
\hline
\end{tabular}

Table 3: BLEU scores for each resource domain on the test set. Here, "BloS", "TedT", "MedW", "WikH" and "OpeS" abbreviate Blogspot, TED-Talks, MediaWiki, WikiHow and OpenSubtitles, respectively.

\begin{tabular}{|c|c|c|c|c|c|c|}
\hline \multirow{3}{*}{ Model } & \multicolumn{6}{|c|}{ Vi-to-En } \\
\hline & $<\mathbf{1 0}$ & {$[10,20)$} & {$[20,30)$} & {$[30,40)$} & {$[40,50)$} & $\geq \mathbf{5 0}$ \\
\hline & $48.77 \%$ & $30.87 \%$ & $13.65 \%$ & $4.46 \%$ & $1.45 \%$ & $0.80 \%$ \\
\hline Google Translate & 32.27 & 34.24 & 37.47 & 38.17 & 37.94 & 38.30 \\
\hline Bing Translator & 34.20 & 32.97 & 36.66 & 38.27 & 39.73 & 40.70 \\
\hline Transformer-base & $36 . \overline{7} \overline{8}$ & $3 \overline{4} . \overline{9} \overline{9}$ & $38 . \overline{2}$ & $\overline{38} .86^{-}$ & $38.76^{-}$ & $39.4 \overline{6}$ \\
\hline Transformer-big & 37.23 & 35.49 & 39.02 & 39.90 & 39.34 & 39.85 \\
\hline mBART & 38.31 & 37.22 & 41.05 & 42.21 & 42.49 & 43.35 \\
\hline
\end{tabular}

Table 4: BLEU scores on the test set w.r.t. sentence lengths of reference English sentences (i.e. the number of word tokens including punctuations). The number right below each length bucket denotes the percentage of sentences belonging to the bucket.

Google Translate and Bing Translator, in which Transformer-big outperforms Transformer-base. In addition, mBART achieves the best performance among all models, reconfirming the quantitative effectiveness of multilingual denoising pre-training for neural MT (Liu et al., 2020).

We present BLEU scores on the Vi-to-En test set for each resource domain and sentence length bucket in Tables 3 and 4, respectively. Table 3 shows that the highest BLEU scores are reported for MediaWiki (wherein documents share and link common events or topics), followed by the ones reported for News and TED-Talks. Three remaining resource domains Blogspot, WikiHow and OpenSubtitles contain less common topic-specific document pairs, thus resulting in lower scores. In Table 4, we find that models produce lower BLEU scores for short- and medium-length sentences (i.e. $<20$ tokens) than for long sentences. This is not surprising as a major proportion of short- and medium- 


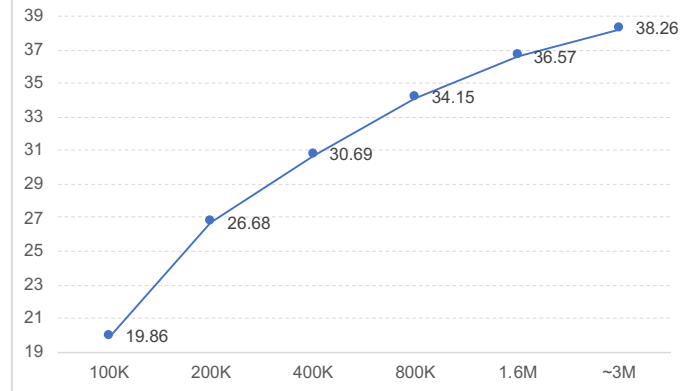

Figure 1: BLEU scores of Transformer-base on the Vito-En validation set when varying training sizes.

length sentences are from OpenSubtitles, while longer sentences generally come from MediaWiki, News and TED-Talks. Note that we observe similar findings for the TER results as well as on the validation set and in the En-to-Vi setup. See additional results w.r.t. each domain and sentence length in the Appendix.

Figure 1 presents BLEU scores of Transformerbase on the validation set for the Vi-to-En setup when varying the numbers of training sentence pairs. Those scores clearly show the effectiveness of larger training sizes.

We also perform an experiment to additionally show that our curation effort has paid off. In particular, as not all of our data are overlapping with OPUS, for a fair comparison, we sample a set of 1.55M non-duplicate Vietnamese-English sentence pairs from OPUS's OpenSubtitles, which has the same size as our PhoMT's OpenSubtitles training subset and do not contain pairs appearing in our OpenSubtitles validation and test subsets. We train two Transformer-base models for Vi-to-En translation: one trained using the sampled OPUS's OpenSubtitles set and another one trained using our OpenSubtitles training subset. Hyper-parameter tuning is performed using our OpenSubtitles validation subset in the same manner as presented in the Appendix. We evaluate the models using our OpenSubtitles test subset. We find that Transformer-base trained using the sampled OPUS's OpenSubtitles set produces a significantly lower Vi-to-En BLEU score on our OpenSubtitles test subset than the one trained using our OpenSubtitles training subset (29.72 vs. 31.11), clearly showing the effectiveness of our quality control. Note that as shown in Table 3, Transformer-base trained using the whole PhoMT's training set obtains a higher Vi-to-En BLEU score at 32.29 on our OpenSubtitles test subset. Thus this experiment also reconfirms the positive effect of a larger training size.

\subsection{Human evaluation results}

We also conduct a human-based manual comparison between the outputs generated by the two automatic translation systems and our three neural baselines. For each translation direction, we randomly sample 100 source sentences in the test set; and for each sentence sample, we anonymously shuffle the translation outputs from five systems. Here, each sampled pair satisfies that any two out of five translation outputs are not exactly the same. We then ask three external Vietnamese annotators to choose which translation they think is the best. ${ }^{4}$ The inter-annotator agreement score computed for Fleiss' kappa coefficient (Fleiss, 1971) between the three annotators is 0.63 which is relatively substantial. Our fourth co-author hosts and participates in a discussion session with the three annotators to resolve annotation conflicts. ${ }^{5}$

Table 2 shows final results, where mBART gains the highest human evaluation scores, thus demonstrating its qualitative effectiveness for both En-toVi and Vi-to-En translation. Table 2 also shows that human preference is not always correlated with the automatic metrics TER and BLEU. For example, in the En-to-Vi setup, though Transformer models have 2+ points better TER and BLEU than Google Translate, they are less preferred by humans than Google Translate (13 vs. 23 and 18 vs. 23). A detailed study is beyond the scope of our paper, but it is worth investigating in future work.

\section{Conclusion}

We have presented PhoMT - a high-quality and large-scale Vietnamese-English parallel dataset of $3.02 \mathrm{M}$ sentence pairs. We empirically conduct experiments on our PhoMT dataset to compare strong baselines and demonstrate the effectiveness of the pre-trained denoising auto-encoder mBART for neural MT in both automatic and human evaluations. We hope that the public release of our dataset can serve as the starting point for further Vietnamese-English MT research and applications.

\section{Acknowledgments}

The authors would like to thank the anonymous reviewers for their helpful feedback.

\footnotetext{
${ }^{4}$ Annotators have a proficient English level at IELTS 7.0+ with a reading score of $8.0+$ (paid 0.25 USD per sample).

${ }^{5}$ The fourth co-author is not involved in both the postprocessing step (Section 2.4) and the development of MT systems. He does not know which model produces which translation. He also has a proficient English level.
} 


\section{References}

Ahmed Abdelali, Francisco Guzman, Hassan Sajjad, and Stephan Vogel. 2014. The AMARA Corpus: Building Parallel Language Resources for the Educational Domain. In Proceedings of the Ninth International Conference on Language Resources and Evaluation, pages 1856-1862.

Željko Agić and Ivan Vulić. 2019. JW300: A WideCoverage Parallel Corpus for Low-Resource Languages. In Proceedings of the 57th Annual Meeting of the Association for Computational Linguistics, pages 3204-3210.

Mikel Artetxe and Holger Schwenk. 2019. Massively multilingual sentence embeddings for zeroshot cross-lingual transfer and beyond. Transactions of the Association for Computational Linguistics, 7:597-610.

Anja Baum. 2020. Vietnam's Development Success Story and the Unfinished SDG Agenda. IMF Working Papers, 20(31):1-31.

Fabienne Braune and Alexander Fraser. 2010. Improved Unsupervised Sentence Alignment for Symmetrical and Asymmetrical Parallel Corpora. In Proceedings of the 23rd International Conference on Computational Linguistics: Posters, pages 81-89.

M. Cettolo, J. Niehues, S. Stüker, L. Bentivogli, R. Cattoni, and Marcello Federico. 2015. The IWSLT 2015 Evaluation Campaign. In Proceedings of the International Workshop on Spoken Language Translation.

Shuoyang Ding, Adithya Renduchintala, and Kevin Duh. 2019. A Call for Prudent Choice of Subword Merge Operations in Neural Machine Translation. In Proceedings of Machine Translation Summit XVII Volume 1: Research Track, pages 204-213.

Ahmed El-Kishky, Vishrav Chaudhary, Francisco Guzmán, and Philipp Koehn. 2020. CCAligned: A Massive Collection of Cross-Lingual WebDocument Pairs. In Proceedings of the 2020 Conference on Empirical Methods in Natural Language Processing, pages 5960-5969.

Joseph L Fleiss. 1971. Measuring nominal scale agreement among many raters. Psychological bulletin, $76(5): 378$.

Armand Joulin, Edouard Grave, Piotr Bojanowski, and Tomas Mikolov. 2017. Bag of Tricks for Efficient Text Classification. In Proceedings of the 15th Conference of the European Chapter of the Association for Computational Linguistics: Volume 2, Short Papers, pages 427-431.

Diederik P. Kingma and Jimmy Ba. 2014. Adam: A Method for Stochastic Optimization. arXiv preprint, arXiv:1412.6980.
Faisal Ladhak, Esin Durmus, Claire Cardie, and Kathleen McKeown. 2020. WikiLingua: A New Benchmark Dataset for Cross-Lingual Abstractive Summarization. In Findings of the Association for Computational Linguistics: EMNLP 2020, pages 4034 4048.

Pierre Lison and Jörg Tiedemann. 2016. OpenSubtitles2016: Extracting Large Parallel Corpora from Movie and TV Subtitles. In Proceedings of the Tenth International Conference on Language Resources and Evaluation, pages 923-929.

Yinhan Liu, Jiatao Gu, Naman Goyal, Xian Li, Sergey Edunov, Marjan Ghazvininejad, Mike Lewis, and Luke Zettlemoyer. 2020. Multilingual Denoising Pre-training for Neural Machine Translation. Transactions of the Association for Computational Linguistics, 8:726-742.

Christopher Manning, Mihai Surdeanu, John Bauer, Jenny Finkel, Steven Bethard, and David McClosky. 2014. The Stanford CoreNLP Natural Language Processing Toolkit. In Proceedings of 52nd Annual Meeting of the Association for Computational Linguistics: System Demonstrations, pages 55-60.

Quoc Hung Ngo, Werner Winiwarter, and Bartholomäus Wloka. 2013. EVBCorpus - A Multi-Layer English-Vietnamese Bilingual Corpus for Studying Tasks in Comparative Linguistics. In Proceedings of the 11th Workshop on Asian Language Resources, pages 1-9.

Dat Quoc Nguyen, Dai Quoc Nguyen, Thanh Vu, Mark Dras, and Mark Johnson. 2018. A Fast and Accurate Vietnamese Word Segmenter. In Proceedings of the 11th International Conference on Language Resources and Evaluation, pages 2582-2587.

Myle Ott, Sergey Edunov, Alexei Baevski, Angela Fan, Sam Gross, Nathan Ng, David Grangier, and Michael Auli. 2019. fairseq: A Fast, Extensible Toolkit for Sequence Modeling. In Proceedings of the 2019 Conference of the North American Chapter of the Association for Computational Linguistics: Demonstrations, pages 48-53.

Myle Ott, Sergey Edunov, David Grangier, and Michael Auli. 2018. Scaling Neural Machine Translation. In Proceedings of the Third Conference on Machine Translation: Research Papers, pages 1-9.

Kishore Papineni, Salim Roukos, Todd Ward, and WeiJing Zhu. 2002. Bleu: a Method for Automatic Evaluation of Machine Translation. In Proceedings of the 40th Annual Meeting of the Association for Computational Linguistics, pages 311-318.

Hong-Hai Phan-Vu, Viet Trung Tran, Van Nam Nguyen, Hoang Vu Dang, and Phan Thuan Do. 2019. Neural Machine Translation between Vietnamese and English: an Empirical Study. Journal of Computer Science and Cybernetics, 35(2):147-166. 
Matt Post. 2018. A Call for Clarity in Reporting BLEU Scores. In Proceedings of the Third Conference on Machine Translation: Research Papers, pages 186191.

Nils Reimers and Iryna Gurevych. 2020. Making Monolingual Sentence Embeddings Multilingual using Knowledge Distillation. In Proceedings of the 2020 Conference on Empirical Methods in Natural Language Processing, pages 4512-4525.

Holger Schwenk, Vishrav Chaudhary, Shuo Sun, Hongyu Gong, and Francisco Guzmán. 2021. WikiMatrix: Mining 135M Parallel Sentences in 1620 Language Pairs from Wikipedia. In Proceedings of the 16th Conference of the European Chapter of the Association for Computational Linguistics: Main Volume, pages 1351-1361.

Rico Sennrich, Barry Haddow, and Alexandra Birch. 2016. Neural Machine Translation of Rare Words with Subword Units. In Proceedings of the 54th Annual Meeting of the Association for Computational Linguistics (Volume 1: Long Papers), pages 17151725.

Rico Sennrich and Martin Volk. 2011. Iterative, MTbased Sentence Alignment of Parallel Texts. In Proceedings of the 18th Nordic Conference of Computational Linguistics, pages 175-182.

Matthew Snover, Bonnie Dorr, Richard Schwartz, Linnea Micciulla, and John Makhoul. 2006. A study of translation edit rate with targeted human annotation. In Proceedings of the 7th Biennial Conference of the Association for Machine Translation in the Americas, pages 223-231.

Jörg Tiedemann. 2012. Parallel Data, Tools and Interfaces in OPUS. In Proceedings of the Eighth International Conference on Language Resources and Evaluation, pages 2214-2218.

Dániel Varga, Péter Halácsy, András Kornai, Viktor Nagy, László Németh, and Viktor Trón. 2007. Parallel corpora for medium density languages. Current Issues in Linguistic Theory, 292:247-258.

Ashish Vaswani, Noam Shazeer, Niki Parmar, Jakob Uszkoreit, Llion Jones, Aidan N Gomez, Ł ukasz Kaiser, and Illia Polosukhin. 2017. Attention is All you Need. In Advances in Neural Information Processing Systems, volume 30, pages 5998-6008.

Thanh Vu, Dat Quoc Nguyen, Dai Quoc Nguyen, Mark Dras, and Mark Johnson. 2018. VnCoreNLP: A Vietnamese Natural Language Processing Toolkit. In Proceedings of the 2018 Conference of the North American Chapter of the Association for Computational Linguistics: Demonstrations, pages 56-60.

Yonghui Wu, Mike Schuster, et al. 2016. Google's Neural Machine Translation System: Bridging the Gap between Human and Machine Translation. arXiv preprint, arXiv:1609.08144.

\section{Appendix}

\section{Parallel document-level corpora}

Last access: 19/12/2020

WikiHow:

- https://github.com/esdurmus / Wikilingua

TED-talks:

- https://object.pouta.csc.fi/ OPUS-TED2020/v1/raw/en.zip

- https://object.pouta.csc.fi/ OPUS-TED2020/v1/raw/vi.zip

MediaWiki:

- https://dumps.wikimedia.org/ other / contenttranslation (version: November 2020)

OpenSub:

- https://object.pouta.csc.fi/ OPUS-OpenSubtitles/v2018/raw/ en.zip

- https://object.pouta.csc.fi/ OPUS-OpenSubtitles/v2018/raw/ vi.zip

News:

- https: / / songngu. dayhoctienganh. net/page/1

- https://toomva.com/ doc-bao-anh-viet $/ \mathrm{pc}=8$ ?page $=0$

- http:// cep.com.vn/news

- https: / / www.hrw. org/languages? language $=$ vi\&page $=0$

- http://vietanhsongngu.com/ hoc-tieng-anh-bai-mau-tintuc-c5.htm

- https://baosongngu.com/ chuyen-muc/world https: //baosongngu.com/ chuyen-muc/vn https: //baosongngu.com/ chuyen-muc/bbc 
- https://www.jw.org/vi/tin-tuc/ phap-ly

https://www.jw.org/vi/tin-tuc/ ve-nhan-chung

https://www.jw.org/vi/tin-tuc/ tin-tuc

Blogspot:

- https://gocsan.blogspot.com

\section{Discussion on the alignment coverage rate}

OPUS (Tiedemann, 2012) also provides a corpus of $350 \mathrm{~K}$ sentence pairs extracted from a newer version of the MediaWiki content translation dump (version: 02 April 2021), ${ }^{6}$ wherein duplicate pair removal is not performed. Note that the number of our MediaWiki sentence pairs is bigger at 500K (without taking duplicate pairs into account), thus again showing that our alignment approach is more effective than the OPUS alignment approach.

\section{Discussion on the use of Google Translate}

To align parallel sentences within a parallel document pair as described in Section 2.3, we first translate each English source sentence into Vietnamese by using Google Translate. Here, the use of Google Translate in this step is via utilizing the "GoogleTranslate" function in Google Sheets. However, we later find that this "GoogleTranslate" function in Google Sheets produces lower performance scores than using the Google Translate API in both automatic and human evaluation setups. Therefore, in our result tables, we report "Google Translate" scores accounted for the Google Translate API on the validation and test sets.

\section{Implementation details}

We employ Transformer and mBART implementations from fairseq (Ott et al., 2019). For both Transformer models (Vaswani et al., 2017), following Ding et al. (2019), we use subword-nmt to learn joint BPE with $32 \mathrm{~K}$ merge operations (Sennrich et al., 2016). For mBART, we finetune the pre-trained sequence-to-sequence model mBART25 (Liu et al., 2020). Here, mBART25 is pre-trained on a Common Crawl dataset of 25 languages, which contains 300GB of English texts and 137 GB of Vietnamese texts. Following Vaswani et al. (2017), we use beam search with a beam size

\footnotetext{
${ }^{6}$ https://opus.nlpl.eu/ wikimedia-v20210402.php
}

of 4 and length normalization of 0.6 for decoding. Due to the model size, we apply batch sizes of 16K tokens for Transformer-base, 8K tokens for Transformer-big and $4 \mathrm{~K}$ tokens for mBART. We optimize the models using Adam (Kingma and Ba, 2014) and run for 30 training epochs, wherein the Adam initial learning rate is warmed up for the first epoch. In addition, we also perform grid search to select the initial learning rate from $\{1 \mathrm{e}-4,3 \mathrm{e}-4,5 \mathrm{e}-$ $4,7 \mathrm{e}-4\}$ for Transformer models and from $\{1 \mathrm{e}-5$, $3 e-5,5 e-5,7 e-5\}$ for mBART. For both Englishto-Vietnamese and Vietnamese-to-English translation setups, the optimal learning rates selected for Transformer-base, Transformer-big and mBART are $5 \mathrm{e}-4,3 \mathrm{e}-4$ and $5 \mathrm{e}-5$, respectively. Here, we evaluate each model 8 times during every training epoch, and then select the model checkpoint that obtains the highest BLEU score on the validation set to apply to the test set.

We compute the detokenized and case-sensitive BLEU score using SacreBLEU (with the signature "BLEU+case.mixed+numrefs.1+smooth.exp+tok.13a+version.1.5.1"). ${ }^{7}$ Similarly, we also compute the detokenized and case-sensitive TER score (with the option "-N" of normalization). ${ }^{8}$

\section{Additional results}

Tables 5 and 6 present details of TER and BLEU scores on the validation and test sets for each domain. In addition, we show TER and BLEU scores for each sentence length bucket in Table 7 and Table 8 , respectively.

\footnotetext{
${ }^{7}$ https://github.com/mjpost/sacrebleu

${ }^{8}$ https://github.com/jhclark/tercom
} 


\begin{tabular}{|c|c|c|c|c|c|c|c|c|c|c|c|c|c|}
\hline \multirow{2}{*}{\multicolumn{2}{|c|}{ Model }} & \multicolumn{6}{|c|}{ En-to-Vi } & \multicolumn{6}{|c|}{ Vi-to-En } \\
\hline & & Tews & BloS & TedT & MedW & WikH & OpeS & News & BloS & TedT & MedW & WikH & OpeS \\
\hline \multirow{5}{*}{ 尝 } & & 5.93 & 7.26 & 49.37 & 2489 & & 60.70 & 2.21 & 51.25 & 46.94 & & 51.08 & 0.29 \\
\hline & & & & & & & & .72 & 52.38 & & & & \\
\hline & & & & & & & & & & & & & \\
\hline & & & & & & & & & & & & & \\
\hline & & .97 & 45.78 & 47.87 & 22.26 & 7.71 & 52.67 & 36.16 & 48.98 & 45.75 & 54 & 01 & 5.56 \\
\hline \multirow{5}{*}{ 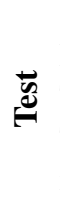 } & & 46 & .05 & 5.45 & 27.0 & 32 & .71 & 9.78 & 56.60 & 45.22 & 8 & 80 & 1.27 \\
\hline & & .44 & & & & & & & 7.89 & 46.95 & & & 9.28 \\
\hline & & 42 & 53.21 & 45.95 & & & & .69 & .64 & 45.84 & & & 47.20 \\
\hline & & & 52.79 & 45 & & & & & & & & & 47.14 \\
\hline & & & & & & 50.01 & 52.44 & 45.86 & 54.10 & 43.57 & 29.90 & 51.21 & 46.16 \\
\hline
\end{tabular}

Table 5: TER results on each domain.

\begin{tabular}{|c|c|c|c|c|c|c|c|c|c|c|c|c|c|}
\hline \multirow{2}{*}{\multicolumn{2}{|c|}{ Model }} & \multicolumn{6}{|c|}{ En-to-Vi } & \multicolumn{6}{|c|}{ Vi-to-En } \\
\hline & & ws & BloS & TedT & MedW & ikH & OpeS & News & BloS & TedT & MedW & WikH & OpeS \\
\hline & & 28 & & 62 & & 60 & .37 & .12 & 85 & 2.21 & & .48 & \\
\hline & & & & & & & & 1.02 & 30.95 & 9.51 & & & 30 \\
\hline & & & & & & & 27.42 & & 30.61 & 30.67 & & & \\
\hline & & & & & & & & & & & & & \\
\hline & & 54.82 & & 34.72 & 67.42 & & 28.18 & .51 & 33.72 & 33.76 & 56.06 & .22 & 4.93 \\
\hline & & & & & & & & & & & & & 3.63 \\
\hline & & & & .58 & & & & & 5.91 & 32.03 & 50.2 & 1.78 & 0.56 \\
\hline & & 89 & 29.54 & 36.72 & 62.69 & .33 & 28.04 & 4.94 & 27.13 & 33.46 & 50.56 & 28.37 & 32.29 \\
\hline & & .33 & 30.37 & 37.66 & 63.58 & 33.31 & 28.58 & 36.14 & 28.26 & 34.46 & 51.2 & 28.86 & 32.69 \\
\hline & mBART & 3.93 & 31.39 & 8.01 & 64.67 & 33.97 & 29.01 & 37.04 & 29.26 & 36.43 & 53.93 & 30.63 & 34.13 \\
\hline
\end{tabular}

Table 6: BLEU results on each domain.

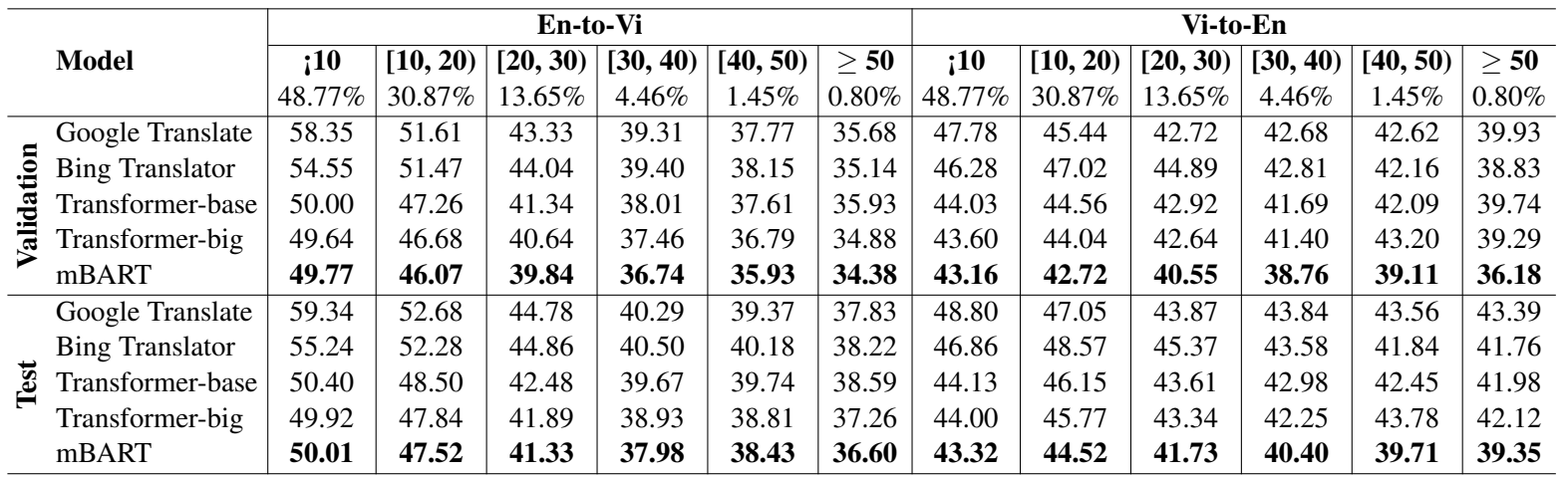

Table 7: TER results w.r.t. sentence lengths of reference English sentences.

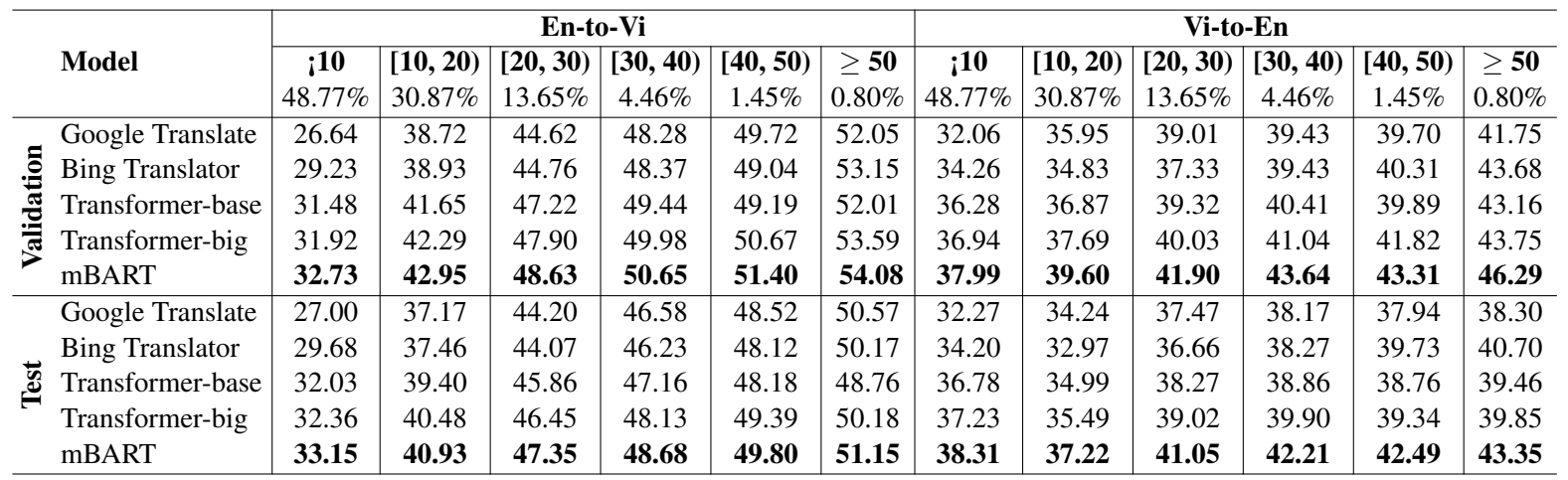

Table 8: BLEU results w.r.t. sentence lengths of reference English sentences. 